\title{
Est-il vraiment pertinent d'évaluer la rédaction du résumé dans le cadre de l'épreuve de lecture critique d'article scientifique?
}

\section{Is it really relevant to assess the written summary aptitude for the scientific article critical reading test?}

Monsieur,

C'est avec plaisir que je lis régulièrement votre journal et j'apprécie que, périodiquement, plusieurs articles soient en lien direct avec les problématiques concrètes auxquelles sont confrontées les facultés de médecine. Il en est ainsi des différentes expériences rapportées concernant l'examen de lecture critique d'article scientifique, tel qu'il est désormais organisé en France à l'issue du deuxième cycle des études médicales, dans le cadre des épreuves classantes nationales ${ }^{[1]}$.

Pour autant, je dois avouer que la lecture de l'article de Muscari et al. ${ }^{[2]}$, qui rapporte l'évaluation de l'utilisation d'une grille enrichie de motsclés pour la correction de la rédaction du résumé, exercice qui constitue une partie l'épreuve, me laisse perplexe. Mes remarques ne visent évidemment ni à contester la légitimité des auteurs à tenter d'apporter une amélioration concernant une épreuve dont la correction a suscitée des réserves, ni à discuter la qualité de leur contribution. Pour qu'il n'y ait aucune ambiguité, je précise aussi d'emblée que je suis un ardent défenseur de la formation à l'analyse d'article scientifique, qui est indispensable pour développer l'esprit critique des futurs médecins et je suis convaincu qu'il convient dès lors que les apprentissages nécessaires soient évalués. Mais on ne peut manquer d'être stupéfait par la somme d'énergie dépensée par les étudiants et les enseignants à propos de cette épreuve du résumé, dont je ne comprends pas l'intérêt, d'autant plus qu'il s'agit de faire le résumé d'un bon article... En l'espèce, on pourrait d'ailleurs considérer que le travail demandé correspond davantage à un exercice de contraction de texte, tels qu'ils sont organisés par exemple dans le cadre de nombreux concours administratifs, qu'à un réel travail d'écriture scientifique.

Depuis 20 ans que j'enseigne la rédaction médicale, comme d'autres enseignants de cette discipline, je dis que faire un bon résumé ne demande aucune imagination car il suffit de copier des phrases de l'article. Il ne faut pas réécrire le travail, ou faire de la paraphrase, car on risque d'introduire des erreurs ou des ambiguïtés par rapport à l'article. À mes yeux, faire un résumé ne demande aucune réflexion car, lorsque l'article original princeps est de bonne qualité, la recette est simple et peut être décrite en quatre étapes :

1) But de l'étude : copier la dernière phrase de l'introduction;

2) Méthodes : copier la première phrase de chaque paragraphe des méthodes, puisque c'est dans ces phrases qu'il y a, en principe, les point principaux des méthodes ; 
3) Résultats : faire, pour chaque tableau ou figure, une phrase qui en résume les données ;

4) Conclusion : copier la première phrase du chapitre discussion (nous avons montré que..., ce qui a changé notre stratégie diagnostique dans cette maladie en... ).

Le « résumé de référence », en annexe 2 de l'article évoqué ${ }^{[2]}$, attire quelques commentaires : dans la section «méthodes », plusieurs phrases concernent en fait des résultats et non pas des méthodes! La conclusion reprend par ailleurs exactement des données du chapitre résultats. Il faut éviter de telles répétitions dans un résumé pour privilégier les informations quand le nombre de mots est limité.

Beaucoup de résumés d'articles scientifiques publiés, y compris dans d'excellentes revues, contiennent des erreurs mais peu de travaux ont été faits sur ce thème, le plus souvent sur de petits échantillons. Il semble que la moitié des résumés d'articles originaux d'essais randomisés contiendraient des erreurs. Dans une étude ${ }^{[3]}, 20$ articles d'essais randomisés publiés en 2005 dans le Journal of the American Medical Association, le New England Journal of Medicine, le British Medical Journal et le Lancet (cinq articles par journal) ont été sélectionnés. Huit résumés structurés sur les 20 analysés contenaient des erreurs en comparaison avec le texte de l'article. Six résumés contenaient des données non présentes dans l'article. Cette étude a été faite sur des résumés publiés dans des journaux qui contrôlent au mieux leurs articles et l'on peut légitimement s'interroger, avec circonspection, sur ce qu'il en est de ceux publiés dans les autres journaux.

Je suis émerveillé par les études montrant que l'épreuve de résumé a un intérêt pédagogique et que la corriger est difficile. Je vous remercie de comprendre que la liberté d'expression dont j'use n'est pas une recherche de polémiques; un débat contradictoire est en général constructif. L'épreuve de lecture d'article est indispensable... mais sans l'épreuve du résumé.

\section{Références}

1. Jouquan J. Quelques questions posées par l'épreuve de lecture critique d'articles scientifiques. Pédagogie Médicale 2005;6:136-7.

2. Muscari F, Montastruc J-L, Fourtanier G. Évaluation d'une grille de correction révisée de l'exercice de rédaction de résumé pour l'examen de lecture critique d'article scientifique dans le cadre des épreuves classantes nationales en France. Pédagogie Médicale 2009;10:19-28.

3. Peacock PJ, Peters TJ, Peacock JL. How well do structured abstracts reflect the articles they summarize? European Science Editing 2009;35:3-5.

Conflits d'intérêts : Directeur Formation médicale continue et médias scientifiques des laboratoires Pfizer; mes opinions n'engagent pas mon employeur.

Hervé Maisonneuve

Président de Global Alliance

for Medical Education, www . game-cme.org Mailto : hervemaisonneuve@gmail.com 